\title{
Prediction of solidification behaviour via microstructure models based on granular structures
}

\author{
A. B. Phillion*1, S. Vernède ${ }^{2}$, M. Rappaz ${ }^{1}$, S. L. Cockcroft ${ }^{3}$ and P. D. Lee
}

Two important factors affecting hot tearing - semi-solid constitutive behaviour and grain percolation - have been simulated through the use of microstructure models based on granular structures. The semi-solid model geometry is based on a modified Voronoi tessellation, and includes rounded corners to approximate an equiaxed-globular grain structure with liquid surrounding the grains. The percolation model combines solidification and thermodynamic aspects to predict the gradual transition within the mushy zone from a continuous liquid to a coherent solid network, while the constitutive behaviour model uses experimentally-derived data to describe the behaviour of the solid grains. By performing a series of models runs over range of grain size and fraction solid, the simulations have revealed an important link between grain size, semi-solid yield stress, strain localisation, and grain coalescence. Furthermore, the models provide insight on the relative importance of each mechanism on hot tear formation, and show promise for improving quantitative hot tearing predictions.

\section{Introduction}

The mathematical computation of solidification behaviour is inherently challenging due to the stochastic distribution of both the liquid phase and the arrangement of the solidifying grains for a given fraction solid. While most previous studies ${ }^{1,2}$ have focused on averaging and macro-scale constitutive models, these studies have had difficulty in reproducing experimentallyobserved defects. For example, the formation of a hot tear has yet to be reliably simulated since, under seemingly identical casting conditions, one billet will crack while another will not. Hot tearing, in which cracks form in the semi-solid region, is an important solidification defect which often leads to rejection of the entire casting. One alternative to the averaging approach is the direct microstructure model based on a granular structure, in which the properties of both the solid and liquid phases can be utilised.

Granular structures have previously been used to model material behaviour during the solidification process. Early models assumed a regular arrangement of grains to describe semi-solid ductility ${ }^{3,4}$ and liquid feeding. ${ }^{5}$ However, this geometry does not approximate solidification very well since the solid grains do not become interconnected until the volume fraction of solid, $g_{\mathrm{s}}=1$.

\footnotetext{
${ }^{1}$ Computational Materials Laboratory, EPF - Lausanne, Switzerland ${ }^{2}$ Melting and Casting Research Unit, Alcan CRV, Voreppe, France ${ }^{3}$ Department of Materials Engineering, University of British Columbia, Vancouver, Canada

${ }^{4}$ Department of Materials, Imperial College London, United Kingdom

*Corresponding author: andre.phillion@epfl.ch
}

Recently, two solidification models have been developed using granular structures based on geometry derived from a Voronoi tessellation. The model by Vernède et al. ${ }^{6,7}$ simulates solidification for globular grains by advancing the grain edges towards the border along a linear segment connecting the nuclei with a Voronoi vertex, taking into account back-diffusion and thermodynamic coalescence at the grain boundaries. Liquid feeding was modelled assuming a Poiseuille flow in each channel, Kirchoff's conservation of flow at the nodal and flow losses compensating solidification shrinkage. Using a similar model, Phillion et al. ${ }^{8}$ predicted the constitutive behaviour of semi-solid globular grains over a wide range of grain size, fraction solid, and percentage porosity. The predictions were made via a direct finite element (FE) simulation of solid grains surrounded by an interconnected liquid. These two models have provided insight into semi-solid percolation and deformation behaviour and have allowed, for the first time, prediction of properties at fraction solid where experiments are not viable and under well defined microstructural configurations.

The goal of the present work is to apply the granular solidification model of Vernède et al. ${ }^{6,7}$ and the granular deformation model of Phillion et al. ${ }^{8}$ to an $\mathrm{Al}-\mathrm{Cu}$ alloy and an $\mathrm{Al}-\mathrm{Mg}$ alloy, in order to determine the effects of cooling rate and grain size on solidification behaviour, and thus to provide greater insight into the mechanisms of hot tearing.

\section{Description of semi-solid granular models}

The two-dimensional granular models used in the current work $^{6,8}$ have been previously, although independently, 


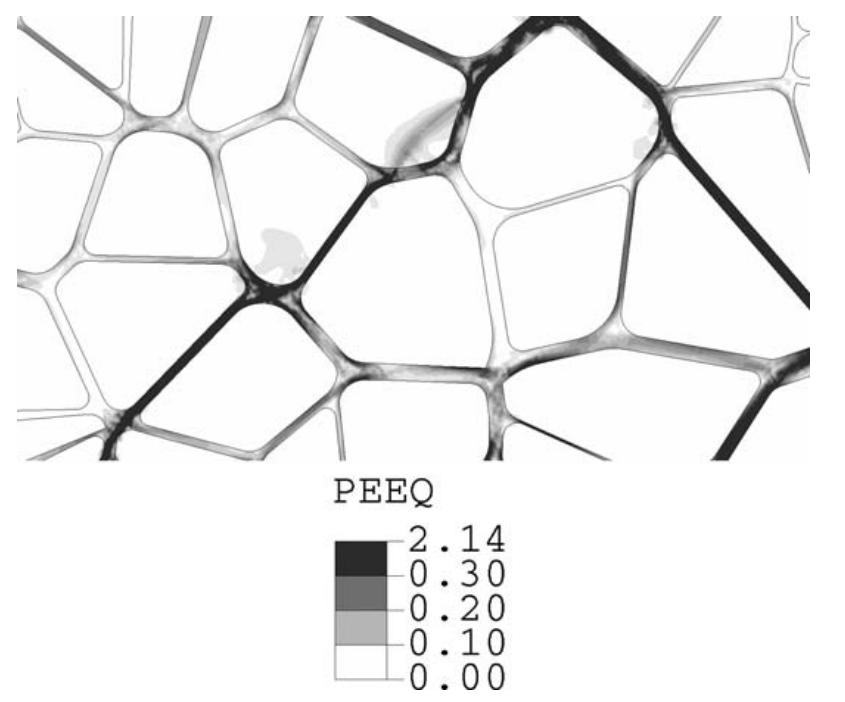

1 Finite element simulation, for $g_{s}=0.85$, showing strain localisation in the liquid and shear banding in the solid

well documented. These microstructural models are based on random nucleation centres in a plane with a given density, and simultaneous grain nucleation. Under these assumptions, the final grain structure will be close to a Voronoi tessellation of the set of nuclei. The sharp grain vertices predicted by the Voronoi tessellation function are then rounded based on a solute flux balance between the Gibbs-Thomson effect and enhanced solute diffusion at the corners. ${ }^{9}$

In the solidification model, each grain is subdivided into triangles formed by connecting the grain nuclei with two Voronoi vertices. The problem is then reduced to one-dimensional solidification in each triangle via a Landu transformation, assuming complete mixing in the liquid phase and back-diffusion in the solid phase. For each increment in fraction solid, the permeability is determined using Darcy's law via a FE calculation of the depression pressure in each of the liquid channels between the grains. The variation in pressure with fraction solid is linked to the variation in velocity of the solid/liquid interface and the liquid channel height.

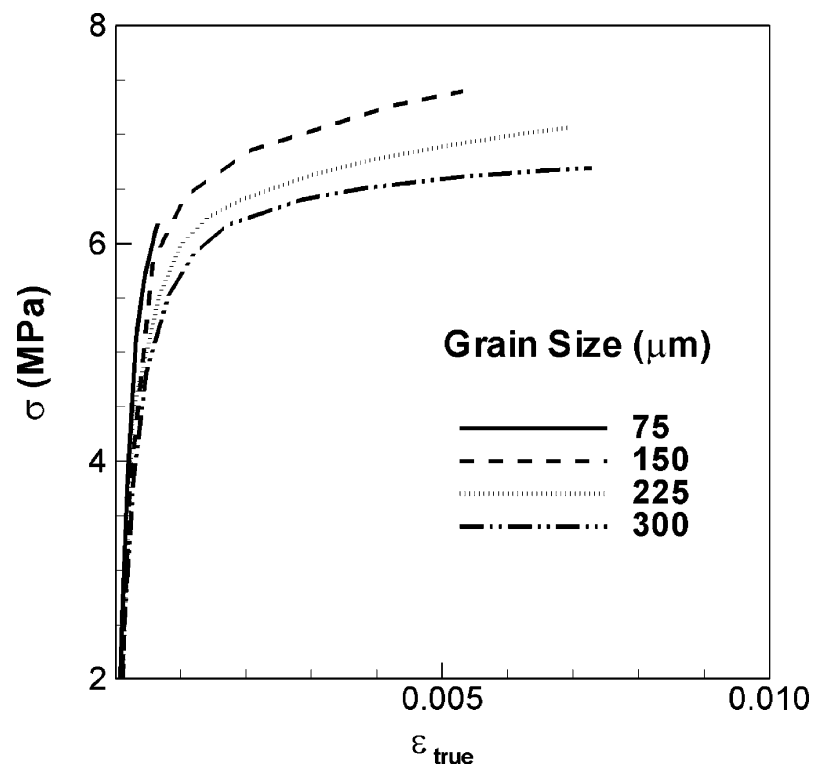

2 The effect of grain size on the predicted semi-solid behaviour, $g_{\mathrm{s}}=0.95$

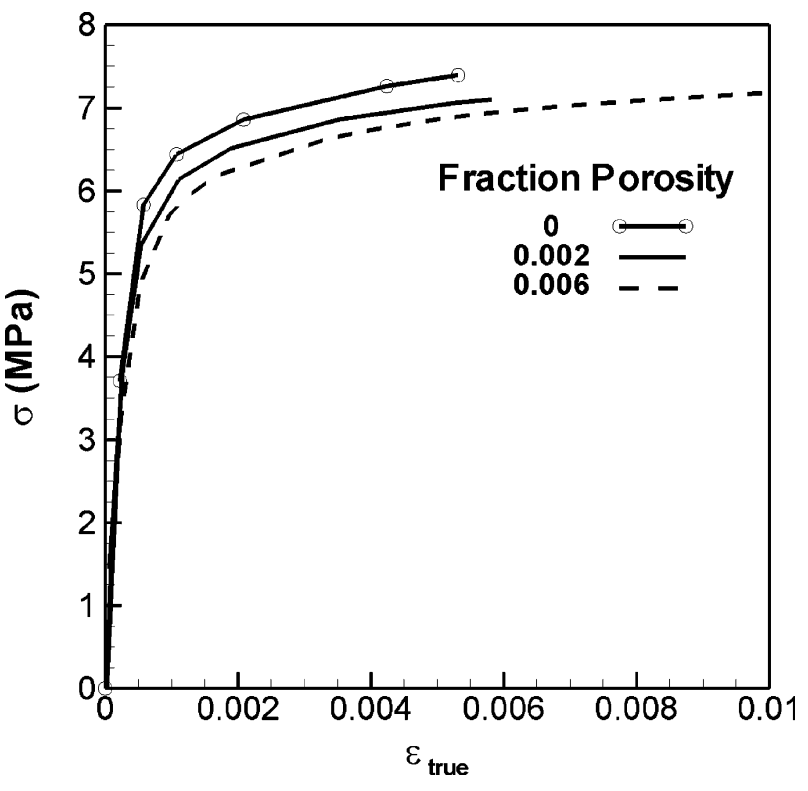

3 The effect of porosity on the predicted semi-solid behaviour, $g_{\mathrm{s}}=\mathbf{0 . 9 5}$

In the semi-solid deformation model, the Voronoi regions are reduced in size via a simple scaling law; the resulting extra material surrounding each region is assumed to be liquid. Both the solid and liquid phases are then meshed using triangular and rectangular elements. An elasto-plastic constitutive equation is used for the solid, while the liquid is approximated as a perfectly plastic material with a very low yield stress. Porosity is introduced by removing liquid material at the Voronoi vertices, i.e. the triple-junctions. The bulk constitutive behaviour is predicted based on the force required to displace the top side of the model domain a distance corresponding to a tensile strain of 0.0225 at a bulk strain rate of $0 \cdot 0015 \mathrm{~s}^{-1}$.

\section{Results and discussion}

The results and discussions pertaining to the two series' of simulations are provided below. The semi-solid deformation model was used to simulate the isothermal

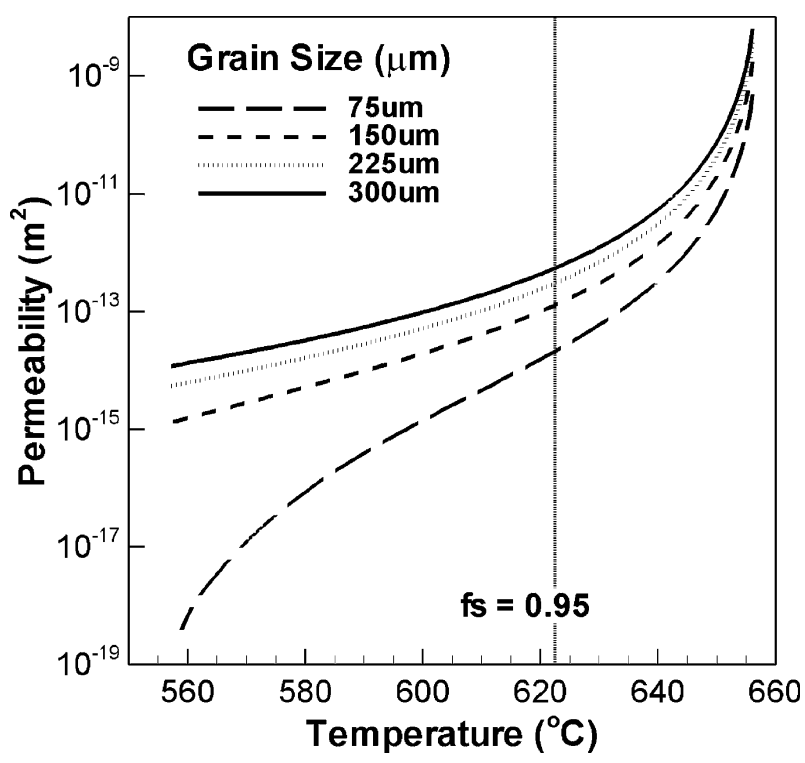

4 The effect of grain size on semi-solid permeability during solidification 




5 The effect of cooling rate on the variation in $g_{\mathrm{s}, \text { perc }}$ as a function of grain size for a simulation of 5000 grains. (a) $300 \mu \mathrm{m}$ (b) $225 \mu \mathrm{m}$ (c) $150 \mu \mathrm{m}$ (d) $75 \mu \mathrm{m}$

tensile deformation in a commercial $\mathrm{Al}-\mathrm{Mg}$ alloy, AA5182, containing 56 grains, for $0.75<g_{\mathrm{s}}<0.95$. Porosity was also included, in amounts less that $0 \cdot 6 \%$ by volume. The solidification model was used to simulate the solidification of an $\mathrm{Al}-1 \mathrm{wt} \% \mathrm{Cu}$ alloy containing 5000 grains, for grain sizes of $75,150,225$, and $300 \mu \mathrm{m}$. For each of the different grain sizes, the cooling rate was varied between 0.004 and $100 \mathrm{~K} \mathrm{~s}^{-1}$. The simulated number of grains is one hundred times larger in the solidification model as compared to the deformation model. This is because the finite element mesh in the solidification model consists of the sub-divided triangles, whereas the element size in the semi-solid deformation model is of the order of $2-5 \mu \mathrm{m}$ in length. In aggregate, the number of degrees of freedom is roughly the same in both models.

\section{Semi-solid deformation model}

The results of the semi-solid deformation simulations, for an AA5182 alloy, are provided in Figs. 1-3. The image in Fig. 1, which is the result of a simulation at $g_{\mathrm{s}}=0 \cdot 85$, shows that the model is able to capture critical semi-solid deformation phenomena: shear banding on some of the grains at higher strain and the accumulation of strain within the liquid. Since the majority of strain is contained within the weak liquid channels, material failure is inevitable.

The effect of grain size on the stress-strain predictions is shown in Fig. 2 for the case with $g_{\mathrm{s}}=0 \cdot 95$. As can be seen in the figure, the flow stress decreases with increasing grain size. Larger grain sizes weaken the semi-solid since they result in thicker liquid channels for a given fraction solid, allowing for increased strain accumulation in the liquid before the solid grains deform. Although not shown in Fig. 2, the effect of grain size decreases with decreasing fraction solid. This is because the relative difference in liquid channel thickness between the large and small grain sizes is significant only at high fraction solid.

The effect of fraction porosity on the stress-strain predictions is provided in Fig. 3 for the cases with 


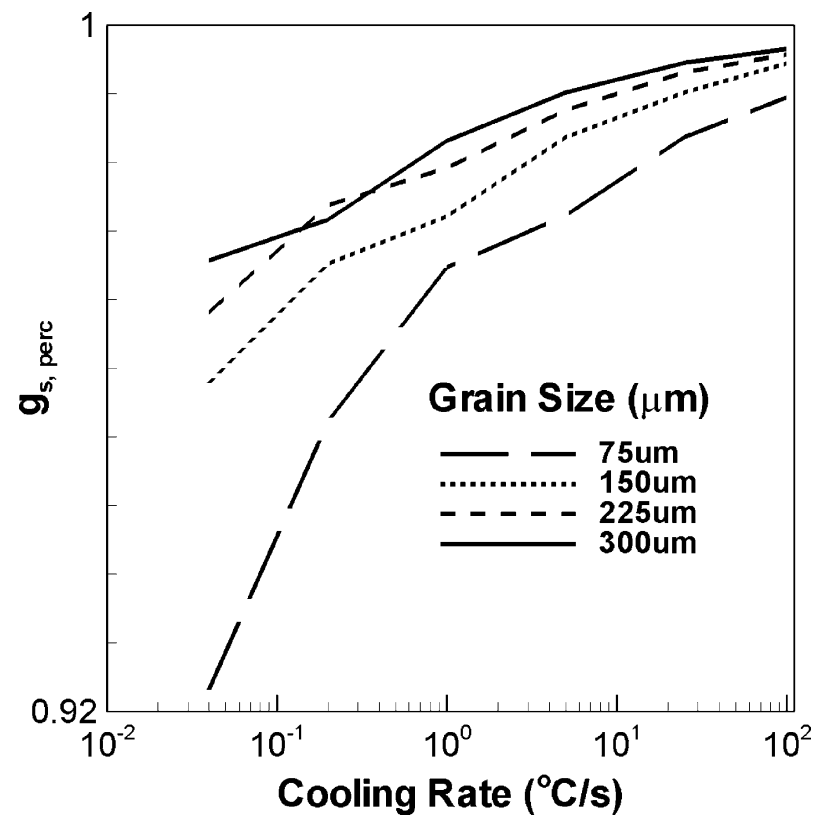

6 Fluid flow through an isothermal mushy zone $\left(3.75 \times 3.75 \mathrm{~mm}^{2}\right)$ for different grain sizes at $g_{\mathrm{s}}=0.975$. The channel widths are proportional to the local flow normalised by the overall flow

$g_{\mathrm{s}}=0 \cdot 95$, and a grain size of $225 \mu \mathrm{m}$. Porosity values of $0,0 \cdot 2$ and $0 \cdot 6 \%$ were investigated. As can be seen in the figure, the addition of porosity to the model geometry results in only a modest decrease in the bulk flow stress. This observation is to be expected for any third-phase particle with a volume fraction which is less than $5 \% .{ }^{10}$ The flow stress decrease is due to changes within the liquid phase since adding voids at the triple-junctions leads to increased strain localisation and deformation. Furthermore, while the fraction porosity is quite small, the voids are placed at critical locations within the geometry and thus can have an important effect on flow stress and ductility.

\section{Solidification model}

The results of the solidification simulations, for an $\mathrm{Al}-$ $1 \mathrm{wt}-\% \mathrm{Cu}$ alloy, are shown in Figs. 4-6. In Fig. 4, the mushy zone permeability has been plotted as a function of temperature for each of the four grain sizes. In Fig. 5, the fraction solid for solid percolation $g_{\text {s,perc }}$ has been plotted as a function of cooling rate for each of the four grain sizes. Percolation is defined as the fraction solid when there is no longer continuity of the liquid path from one side to the other side of the model domain. Thus, many of the grains are in mechanical contact and the remaining liquid exists as isolated pockets only. In Fig. 6, the normalised fluid flow through an isothermal mushy zone at $g_{\mathrm{s}}=0.975$ is shown for a series of microstructures with different grain sizes but a fixed domain size.

Beginning with Fig. 4, it can be seen that the mushy zone permeability decreases with decreasing temperature, due to the increasing fraction solid and thus the increasing resistance to liquid flow. Furthermore, the effect of grain size on permeability is significant when the last liquid undergoes solidification, as larger permeability is predicted for larger grain sizes. For example, the permeability at $560^{\circ} \mathrm{C}$ is $\sim 1 \times 10^{-14}$ and $\sim 6 \times 10^{-19}$ for grain sizes of 300 and $75 \mu \mathrm{m}$. The argument is often made that hot tearing occurs because of limited semisolid permeability, which impedes liquid flow to counteract tensile deformation. The permeability comparison of Fig. 4 is thus surprising, since materials with smaller grain size are known to be less susceptible to hot tearing. However, the size of the domain must also be taken into account due to the localised feeding through larger channels. The computations for Fig. 4 were done for a constant number of grains and thus a varying domain size.

Fig. 6 shows pictorially the effect of grain size on fluid flow for a fixed domain size. It is clear that when domain size is included, the actual number of liquid channels available for fluid flow becomes an important factor. In Fig. 6a, $300 \mu \mathrm{m}$, the majority of the liquid flow occurs through one channel only. The number of dominant channels is 2 for $225 \mu \mathrm{m}, 3$ for $150 \mu \mathrm{m}$, and $>4$ for $75 \mu \mathrm{m}$. Thus, although the permeability is much lower for the $75 \mu \mathrm{m}$ case (Fig. 6d), the ability to move liquid through different channels in response to a tensile stress improves an alloy's resistance to hot tearing. In the case of $300 \mu \mathrm{m}$ grain, the level of tensile deformation predicted in Fig. 1 will localise strain due to the reduced number of liquid channels, and lead to material failure.

While semi-solid permeability is one aspect of the mushy zone morphology, a second important aspect is the fraction solid for percolation of the solid phase, $g_{\text {s,perc }}$. As can be seen in Fig. $5, g_{\text {s,perc }}$ is dependent on both cooling rate and grain size. First, smaller grains have lower values of $g_{\text {s,perc }}$. Second, the results indicate that $g_{\mathrm{s} \text {,perc }}$ increases with increasing cooling rate. Third, the effect of grain size on $g_{\text {s,perc }}$ is significant for low cooling rates, but decreases in importance for increasing cooling rates. Since percolated semi-solid materials are able to resist the tensile deformations that lead to hot tearing, castings with a lower value of $g_{\mathrm{s} \text {, perc }}$ will tend to have improved hot tearing resistance. This is because the critical temperature range for hot tearing will be reduced. Based on the results of Fig. 5, castings with low cooling rates and small grain sizes should exhibit this improved behaviour. Unfortunately, these two parameters are opposite since low cooling rates give rise to large grains, and conversely, small grains are generally a sign of high cooling rates unless the casing is inoculated using grain refiner or subjected to magnetic stirring.

\section{Conclusions}

Granular solidification models offer new and interesting features for predicting solidification behaviour. On the basis of the above granular solidification and semi-solid deformation simulations, the following conclusions can be summarised:

1. Semi-solid flow stress decreases with both increasing grain size and increasing fraction porosity due to strain localisation within the liquid.

2. Semi-solid permeability decreases with decreasing grain size.

3. The fraction solid for percolation, $g_{\mathrm{s} \text {, perc }}$, decreases with decreasing grain size and decreasing cooling rate.

Based on these results, it is clear that hot tearing susceptibility will be reduced for material containing small grain sizes since this will result in a higherstrength semi-solid material and more liquid channels 
to distribute the tensile deformation while at the same time reducing the solidification window when strain is able to localise in the liquid before solid percolation.

\section{Acknowledgements}

The authors would like to thank NSERC, EPSRC (GR/ T26344), and Alcan CRV (France) for funding this research.

\section{References}

1. DG Eskin, Suyitno, and L Katgerman: Prog. Mater. Sci., 2004, 49, 629-711.
2. PD Lee, A Chirazi, and D See: J. Light Metals, 2000, 1, 15-30.

3. DJ Lahaie and M Bouchard: Metall. Mater. Trans. B, 2001, 32, 697-705.

4. D Larouche, J Langlais, $\mathrm{W} \mathrm{Wu}$, and $\mathrm{M}$ Bouchard: Metall. Mater. Trans. B, 2006, 37B, 431-443.

5. WO Dijkstra, C Vuik, AJ Dammers, and L Katgerman: 'Network modeling of liquid metal transport in solidifying aluminium alloys', Solidif Proc \& Microst: Symp in honor of W Kurz, TMS, 2004, $151-156$.

6. S Vernede and M Rappaz: Acta Mater., 2007, 55, 1703-1710.

7. S Vernede, P Jarry, and M Rappaz: Acta Mater., 2006, 54, 40234034.

8. A. B. Phillion, S. L. Cockcroft and P. D. Lee: Acta Mater., 2008, 56, 4328-4338.

9. M Rappaz, A Jacot, and WJ Boettinger: Metall. Mater. Trans. A, 2003, 34, 467-479.

10. JA Eady and DM Smith: Mater. Forum, 1986, 9, 217. 